\title{
REVIEW \\ Microguards and micromessengers of the genome
}

\author{
D Green ${ }^{1}$, T Dalmay ${ }^{2}$ and T Chapman ${ }^{2}$
}

The regulation of gene expression is of fundamental importance to maintain organismal function and integrity and requires a multifaceted and highly ordered sequence of events. The cyclic nature of gene expression is known as 'transcription dynamics'. Disruption or perturbation of these dynamics can result in significant fitness costs arising from genome instability, accelerated ageing and disease. We review recent research that supports the idea that an important new role for small RNAs, particularly microRNAs (miRNAs), is in protecting the genome against short-term transcriptional fluctuations, in a process we term 'microguarding'. An additional emerging role for miRNAs is as 'micromessengers'-through alteration of gene expression in target cells to which they are trafficked within microvesicles. We describe the scant but emerging evidence that miRNAs can be moved between different cells, individuals and even species, to exert biologically significant responses. With these two new roles, miRNAs have the potential to protect against deleterious gene expression variation from perturbation and to themselves perturb the expression of genes in target cells. These interactions between cells will frequently be subject to conflicts of interest when they occur between unrelated cells that lack a coincidence of fitness interests. Hence, there is the potential for miRNAs to represent both a means to resolve conflicts of interest, as well as instigate them. We conclude by exploring this conflict hypothesis, by describing some of the initial evidence consistent with it and proposing new ideas for future research into this exciting topic.

Heredity (2016) 116, 125-134; doi:10.1038/hdy.2015.84; published online 30 September 2015

\section{INTRODUCTION}

Small RNAs, RNA silencing and microRNA biogenesis

Small RNAs are a diverse set of functional, non-coding RNA molecules that are key regulators of expression for many genes in the genome via the process of gene silencing (Bartel and Chen, 2004; Hall and Dalmay, 2013). Initially, 'small RNA' referred to any class of non-coding RNA molecule 50-250 nucleotides (nt) in length. However, over the last $10-15$ years, the term is more often used to define 'smaller' small RNAs of 19-32 nt, including small interfering RNAs, piwi-associated RNAs and the microRNAs (miRNAs) on which we focus in this review (Perkel, 2013; Figure 1). Small RNAs are produced via the processing of longer precursor RNAs. These precursors can be transcripts of small RNA genes, pre-messenger RNAs (from which introns are released during splicing and processed into small RNAs), tRNAs, small nucleolar RNAs and YRNAs. RNA polymerase II selectively transcribes mRNAs and most miRNA precursors, whereas other small RNAs are derived from housekeeping loci that are transcribed by RNA polymerase III. Small RNAs may be cleaved in a sequence- and structure-specific manner from parent RNAs such as YRNAs and tRNAs, with the latter tRNA-derived small RNAs then entering RNA-mediated gene silencing pathways (Kumar et al., 2014). Research into small RNAs is currently proceeding at an astonishing rate, with ever-increasing knowledge of the discovery of the functions of the various classes of small RNAs as well as the pathways they utilise to alter gene regulation.

The huge interest in small RNAs is because of their roles in gene silencing through RNA-mediated mechanisms (Ghildiyal and Zamore, 2009). RNA silencing is an umbrella term for all small RNA-mediated inhibition of transcription, translation and deactivation of transposable elements. RNA silencing is widely regarded as a master controller of gene regulation. However, small RNAs are known to have important roles in an increasing variety of eukaryotic biological processes. For example, small RNAs have recently been discovered to have a role in transgenerational inheritance and epigenetic memory (Rechavi et al., 2014).

We focus here on the most prominent and well-studied class of eukaryotic small RNAs, the miRNAs, which have diverse cellular roles but are best known for silencing and fine-tuning the expression of mRNA transcripts. miRNAs are evolutionarily conserved and can silence one to many hundreds of mRNA targets (Bartel and Chen, 2004; Kim et al., 2009). In the canonical biogenesis pathway, miRNA genes are transcribed by RNA polymerase II (Figure 2). The primiRNA transcript is cleaved by Drosha/DGCR8 (or Drosha/Pasha in Drosophila melanogaster fruit flies). The subsequent $\sim 70 \mathrm{nt}$ premiRNA forms a characteristic hairpin secondary structure, which is vital for enabling export from the nucleus by RanGTP-dependant nuclear envelope-bound Exportin-5 (Filipowicz et al., 2008; Lee et al., 2004). Dicer, an RNase III/helicase multi-domain enzyme, processes the pre-miRNA into a $\sim 22$ bp miRNA-5p and miRNA-3p duplex. The pair is unwound with one mature miRNA strand selected by the Ago2/ Piwi effector structure of the RNA-induced silencing complex. RNAinduced silencing complex uses the miRNA sequence as a guide for targeting complementary sequences in the mRNA 3'-untranslated region, also known as the miRNA recognition element. In animals, mRNA 5'-untranslated regions have recently been discovered to also contain miRNA recognition elements (Zhou and Rigoutsos, 2014).

${ }^{1}$ Norwich Medical School, University of East Anglia, Norwich Research Park, Norwich, UK and ${ }^{2}$ School of Biological Sciences, University of East Anglia, Norwich Research Park, Norwich, UK

Correspondence: Professor T Chapman, School of Biological Sciences, University of East Anglia, Norwich Research Park, Norwich NR4 7TJ, UK.

E-mail: tracey.chapman@uea.ac.uk

Received 20 March 2015; revised 23 July 2015; accepted 3 August 2015; published online 30 September 2015 


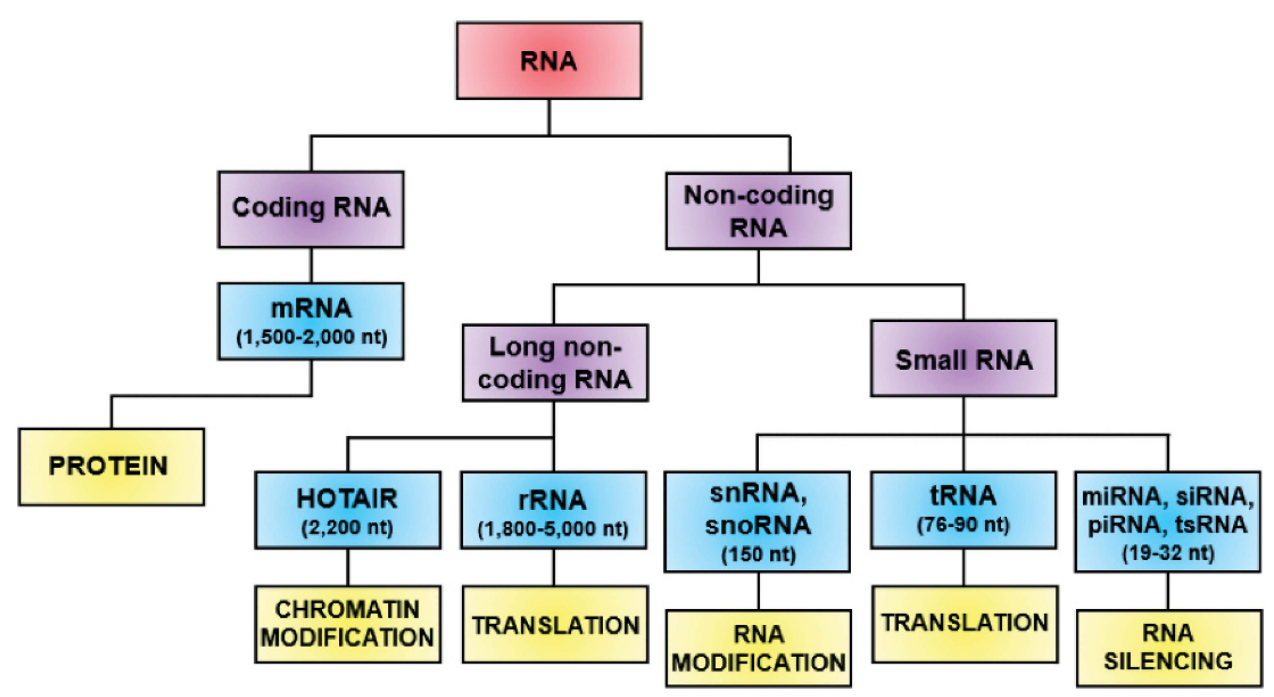

Figure 1 Eukaryotic RNA nomenclature and function. The small RNA family: small RNAs were initially defined as non-coding RNA molecules of 50-250 nucleotides (nt). However, with the discovery of a plethora of 19-32 nt non-coding families of RNAs over the last two decades, the term is now generally used to describe these 'smaller' small non-coding RNAs (Perkel, 2013).

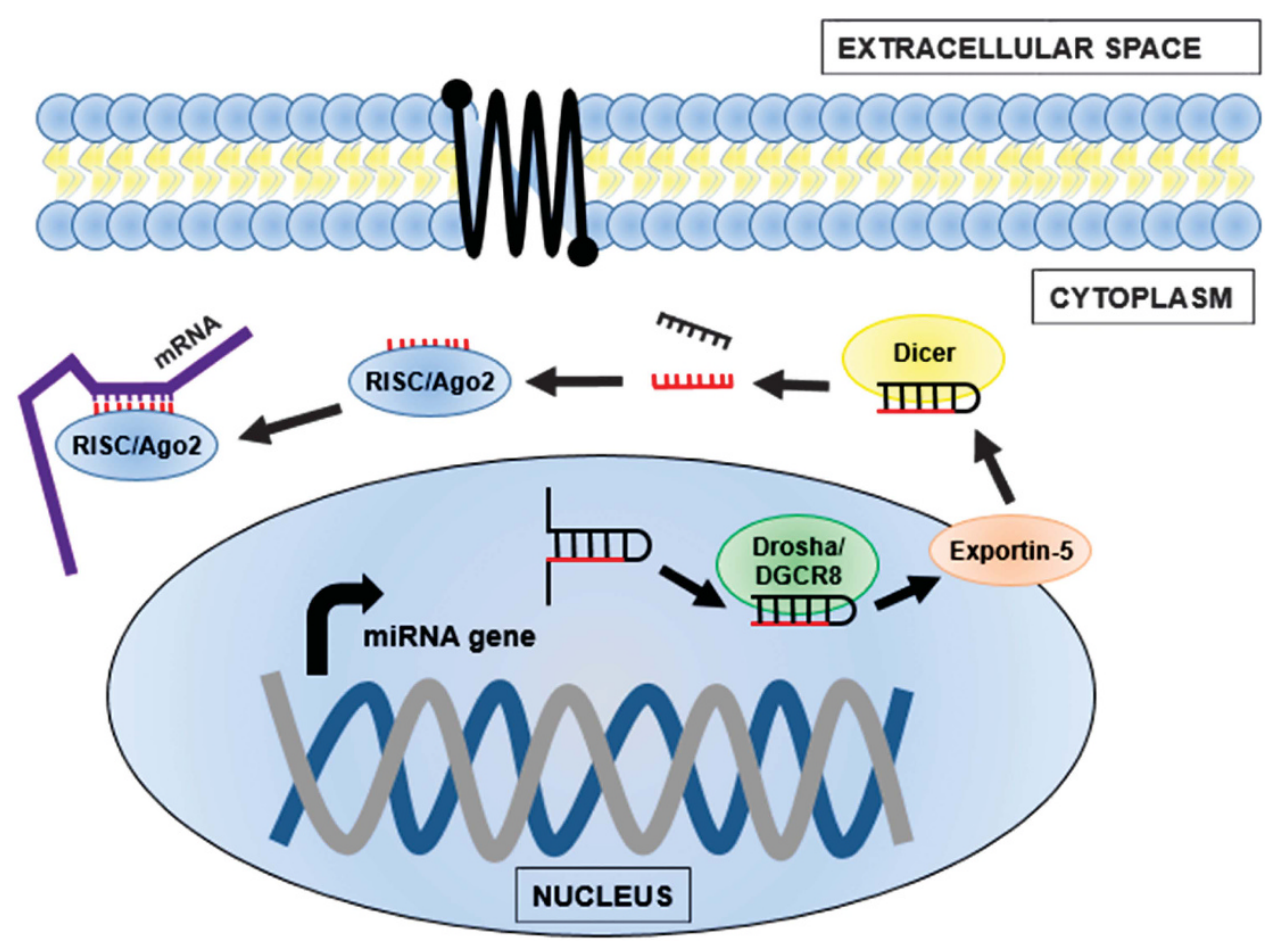

Figure 2 Biogenesis and mode of action of miRNAs. Pri-miRNA genes are transcribed from the genome by RNA polymerase II. The initial transcript is processed to become a shorter pre-miRNA by the microprocessor complex, a protein assembly of an RNase III enzyme known as Drosha and its RNA-binding partner, DGCR8 (also known as Pasha in fruit flies and worms). Exportin-5 facilitates the transport of the pre-miRNA into the cytoplasm. Here, the premiRNA undergoes further processing by an RNase III/helicase enzyme, Dicer, to become a $\sim 22$ bp miRNA duplex. The mature single-stranded miRNA is loaded into the RISC, which uses the miRNA sequence as a guide to bind complementary targets. Gene expression is modified by inhibition of ribosome loading, prevention of eukaryotic initiation factor function and mRNA cleavage.

Accordingly, a gene is silenced or regulated via mRNA translational suppression, mRNA degradation or mRNA cleavage. The importance of miRNAs in gene regulation is illustrated by the evidence that they can regulate up to two-thirds of human genes (Hall and Dalmay, 2013). Gene regulation by miRNAs is of key importance in many fundamental biological processes such as cellular differentiation, proliferation, migration and apoptosis. Dysregulation of miRNA expression has been identified in many diseases and disorders (Dalmay, 2008; Swingler et al., 2012). Therefore miRNAs are also promising biomarkers and potential treatment targets in genetic medicine.

In this review we focus on two new functions for miRNAs-their role as both guards against, and instigators of, gene expression variation. We first describe the evidence to support the idea that 
miRNAs can act as 'microguards' of the genome and second that they can also be trafficked to, and alter gene expression in, target cells and hence function as 'micromessengers'. We conclude by proposing that these two types of functions are likely to become the subject of conflicts of interest in the frequent situations in which interactions occur between cells with low relatedness (for example, different cell lineages, individuals or species). We review the evidence that is consistent with this hypothesis and propose further work to explore this exciting new possibility.

\section{miRNAs as molecular guards}

Initially, much research focussed on understanding the 'on/off' effects of miRNAs. Although this is crucial for understanding the functions and targets of miRNAs, it overlooks the role and key importance of miRNAs in the continual fine-tuning of gene expression to maintain organismal function (Kim et al., 2013). Organisms have adapted to survive in the face of short-term variation in extrinsic (nutrition, temperature) and intrinsic (reproductive) stresses. However, continual bombardment from damaging exogenous factors may overwhelm stress protection responses and decrease fitness. New research proposes that this kind of 'genetic inflammation' can be minimised by internal buffers in the form of miRNAs. Recent findings suggest that in organisms undergoing rapid change-for example, during development or in response to periodic external perturbations such as mating-miRNAs can switch from gene regulators to molecular guards (Fricke et al., 2014; Kim et al., 2013). In this latter role, miRNAs can buffer against abrupt fluctuations in mRNA transcription. This can be important to prevent deleterious effects of variation in mRNA transcript abundance and hence minimise genetic instability leading to a loss of fitness (see below). This ephemeral guarding mechanism is thought to be distinct from the sustained regulation of genes during longer-lasting, cyclical transcription dynamics (Hager et al., 2009). Several examples of the microguarding phenomenon in different contexts are given below.

Microguarding to buffer fluctuations in gene expression during development. In the nematode worm Caenorhabditis elegans (and presumably many other organisms) thousands of transcripts either oscillate according to the different phases of larval development or show temporal gradients of increasing or decreasing expression (Kim et al., 2013). The correct marshalling of these complex expression patterns is necessary for successful development. It has been shown that one mechanism by which a stable, increasing temporal gradient of an important developmental transcription factor known as lin-14 can be achieved against this background of developmental oscillations is through the pulsatile expression of miRNA lin-4 that targets it (Kim et al., 2013). Synchronisation of the profile of expression of the miRNA against the background therefore results in a dampening of the oscillation of its target mRNA. That there has been natural selection to maintain this delicate balance and the microguarding potential of the miRNA is shown by the fact that a failure to correctly balance or integrate these signals can lead to fluctuations in mRNA transcription resulting in developmental arrest (Kim et al., 2013). This example of a 'miRNA-mediated incoherent feed-forward loop' reveals microguarding as a protective genetic layer against imbalances in postembryonic developmental gene expression.

Microguarding to buffer against interactions with the opposite sex. Reproduction involves a complex series of choreographed events that must occur in the correct sequence in order for eggs to be produced, fertilised and laid/implanted. In invertebrates, many of the reproductive events that occur in females during and after mating are initiated by seminal fluid molecules transferred along with sperm during mating (Sirot et al 2014). In D. melanogaster there are $>130$ such proteins and peptides that cause striking changes to egg production, ovulation, female sexual receptivity, immune genes and sleep patterns (reviewed by Sirot et al., 2014). Consistent with this, the receipt of seminal fluid proteins causes widespread, rapid gene expression (for example see Lawniczak and Begun 2004; McGraw et al., 2004, 2008; Gioti et al., 2012) and physiological changes (Sirot et al., 2014) in females. The stimulation of this diverse suite of reproductive processes in females is not without potential cost and receipt of elevated levels of seminal fluid proteins causes reduced female lifespan and reproductive success (Chapman et al 1995; Wigby and Chapman, 2005). These effects are partly due to the actions of a specific seminal fluid protein known as the sex peptide (Chapman et al., 2003b; Liu and Kubli 2003). The underlying genomic signatures of sex peptide receipt are evident in females in diverse changes in many mRNAs over time and in different body parts (Gioti et al., 2012).

Post-mating responses initiated by one sex therefore lead to widespread changes in gene expression in the other (for example see Gioti et al., 2012; Sirot et al., 2014). There are many situations in which reproduction has the potential to cause unwanted fluctuations in gene expression leading to fitness loss for the focal individuals involved (for example see Wigby and Chapman, 2005). We therefore expect selection to buffer against the potentially deleterious matinginduced fluctuations in gene expression.

Consistent with this idea, the expression of mating-responsive miRNAs in females can protect against the sustained deleterious effects of reproduction-induced transcriptional changes (Fricke et al., 2014; Figure 3). This conclusion was reached because females lacking specific miRNAs showed 'unbuffered phenotypes'. For example, females that are hypomorphic for miR-279 (and/or potentially miR-996, see Sun et al., 2015) and miR-317 showed higher reproductive output following mating than controls. Given that elevated reproductive output is accompanied by costs to females, the endogenous level of expression of these miRNAs may act to reduce reproductive output and hence minimise any potential costs. These miRNAs appear to function in this context to block against increased short-term, mating-induced increases in reproductive output, in order to maintain female fitness and extend reproduction over a longer time scale. Similarly, females lacking miR-279 (and/or miR-996, Sun et al., 2015) and miR-317 also tended to re-mate more readily than controls, which suggests that the normal wild-type level of expression could serve to limit over-frequent and costly matings (Chapman et al., 1995, 2003a). By expressing these miRNAs the female can potentially safeguard her genome against deleterious gene expression fluctuations arising from reproduction-related energy expenditure. The results were also consistent with the idea that continual exposure to the exogenous stress of mating with males is more costly to females when miRNA levels were reduced. Specifically, females lacking the miR-278 locus had significantly reduced lifespan upon continual exposure to males than controls. Therefore, the wild-type level of miR-278 was apparently protective against female loss of life. Microguarding in these examples appears to modify genomic responses to exogenous reproductive signals to prevent genome instability, accelerated ageing and an early demise.

Microguarding against the effects of infection. The deleterious consequences for host fitness of perturbation of gene expression arising from infection are widespread. We would again expect selection for 


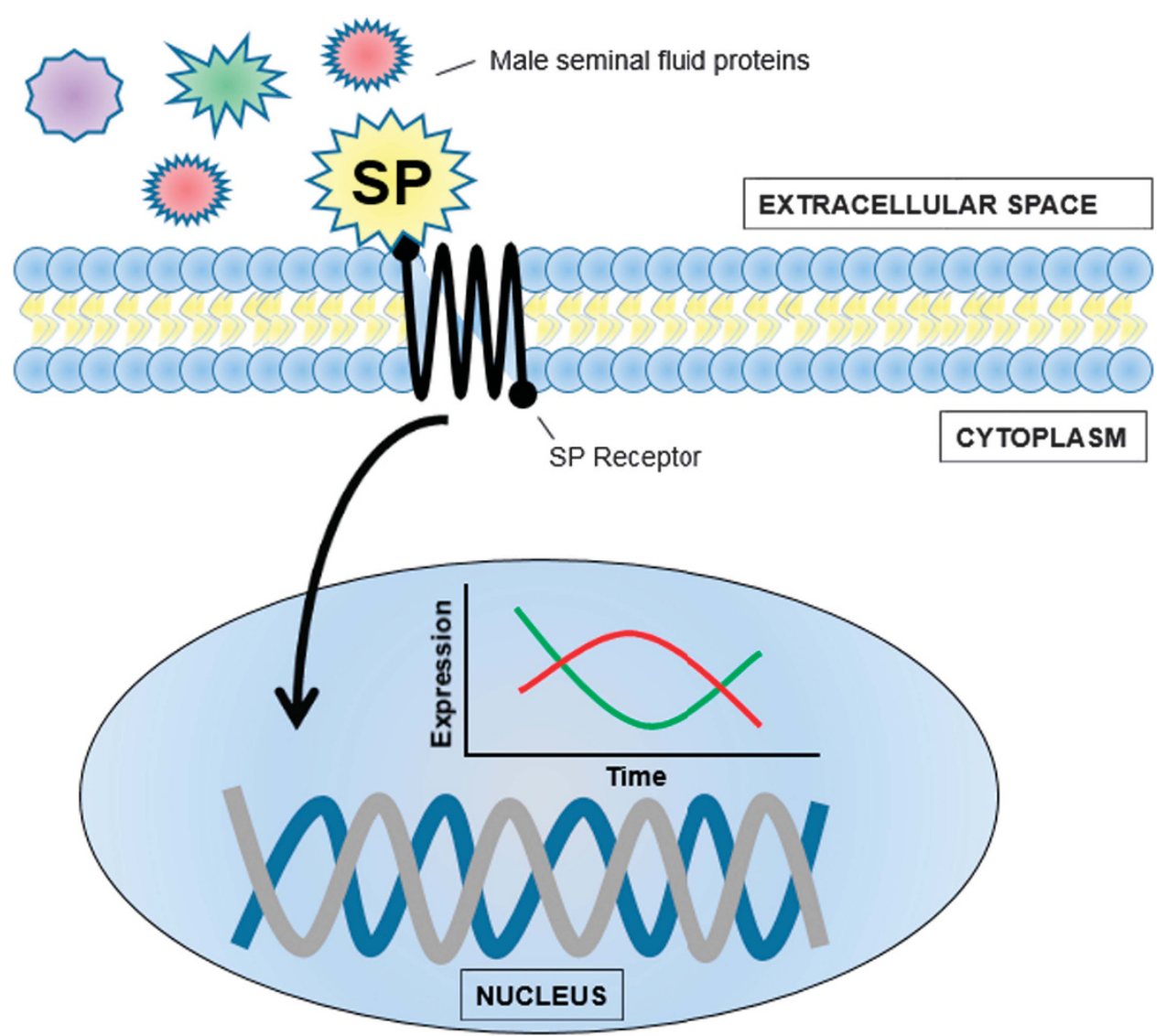

Figure 3 An example scheme for microguarding, using the fruit fly example. Sex peptide (SP), one of $\sim 130$ male seminal fluid proteins, is transferred into the female during mating and activates the SP receptor in the female genital tract and central nervous system. The activated SP receptor initiates intracellular signalling that alters the transcription of genes involved in behaviour, embryology, immunity and ageing. In this hypothetical model, deleterious fluctuations of mRNA expression in the female (red), facilitated by receipt of male ejaculate proteins, are potentially smoothed and dampened by synchronous expression of miRNAs by females (green) as a potential defence mechanism to reduce costs.

microguards to reduce the deleterious impact of such fluctuations. Such an example comes from the study of human infection. Inflammation is a carefully choreographed immune response to a site of contagion, with toxic molecules and chemokines released into the microenvironment. Although this is lethal to invading pathogens, it is also harmful to surrounding healthy tissue. Therefore, inflammatory responses are usually short lived. It is well-recognised that chronic inflammation can increase the risk of age-related diseases such as cancer and musculoskeletal disorders. Under sustained inflammation, mechanisms that limit and protect the body against the deleterious side effects may break down. In an experiment on human cells, a proinflammatory environment was found to induce the increased expression of miR-155 (Tili et al., 2011). A target transcript of this miRNA is the serine/threonine-specific protein kinase WEE1, a cell cycle inhibitor and tumour suppressor gene. Other miR-155 targets included DNA mismatch-repair genes: creating a downward spiral of further mutation rates in the purine salvage enzyme hypoxanthineguanine phosphoribosyltransferase. External interference to microguarding is analogous to chronic inflammation. A re-balancing of this system may offer a new approach for studying and treating such disorders.

The examples listed above may represent just the tip of the iceberg and much more research is clearly needed to discover how widespread the phenomenon of microguarding might be. We need further fitness tests of the consequences of the lack of buffering functions across many different contexts and to better understand the selective forces that will promote microguarding function. However, the examples show proof of principle that the absence or over-abundance of microguarding may result in significant fitness costs. Hence there are significant potential benefits associated with the ability to microguard in response to potentially diverse sources of perturbation.

miRNAs as molecular messengers with the potential to alter gene expression

Cell-to-cell communication can occur via direct contact or by the actions of molecules that are secreted into the extracellular space to reach local or distant targets. There has been huge recent interest in the role of miRNAs in extracellular communication. The big surprise has been the discovery that extracellular miRNAs can remain stable, and be transported, if they are packaged in microvesicles (MVs). Hence miRNAs can, in principle, retain their biological functionality to regulate gene expression in cells distant from their site of synthesis (Valadi et al. 2007). This has given rise to a new and exciting hypothesis that miRNAs may mediate an important new mechanism of cell-cell communication (Valadi et al. 2007; Wang et al., 2010). Hence, along with the potential for miRNAs to function as microguards as described above, that they may also be transported within and between individuals as micromessengers. 
The initial interest in miRNAs as micromessengers was stimulated by the finding that miRNAs can be found at appreciable levels within extracellular MVs (Valadi et al., 2007). miRNA-containing MVs are now known to found in many different biofluids such as plasma, breast milk and semen (Vojtech et al., 2014) and can be released by many cell types (Lotvall and Valadi, 2007). Small RNA and mRNA trafficking first achieved widespread recognition in plants (Yoo et al., 2004; Marin-Gonzalez and Suarez-Lopez, 2012; Parent et al., 2012) and has now also been recognised as potentially widespread in animal cells (Valadi et al., 2007).

However, in order for miRNAs to be molecular messengers with the capacity to be trafficked to remote sites in animals, suitable transport vehicles are required for stability and transport. For example, RNAs need to be protected against degradation by RNAses in the extracellular environment (Chen et al 2008; Mitchell et al. 2008). Valadi et al. (2007) first reported that miRNAs could be packed into MVs and also demonstrated proof of principle that $\mathrm{MV}$-associated RNA could be transferred into recipient cells. miRNAs, as well as mRNAs, proteins and DNA can be found within a variety of different types of MVs that are released from cells into the extracellular environment (Mathivanan et al., 2010; reviewed by Weilner et al., 2013).

MVs are diverse and are generally classified on the basis of size, shape, composition and origin. The classification of MV types remains complex-however, there are generally thought to be three types: ectosomes, exosomes and apoptotic bodies. Ectosomes bud off from the plasma membrane, exosomes are much smaller MVs and formed from vesicles within the cell that can then fuse with the plasma membrane and apoptotic bodies pinch off from the plasma membrane. Though the characteristics of the different types vary, all of them have been found to have the capacity to contain miRNAs once they emerge into the extracellular environment (Weilner et al., 2013). miRNAs can certainly be found inside MVs, but may also adhere to the MV membrane. What is not yet clear however, is whether the concentrations of miRNAs within any/some/all of the various types of MVs are always sufficient to effect gene silencing in target cells (Skog et al., 2008; Chevillet et al., 2014). That said, non-conventional roles for miRNAs might be effected at lower concentrations, a possibility that requires further testing. In this review we refer to MVs as a whole, but recognise that there is huge complexity within this class of biological transport vehicles, whose significance in this context is far from being understood.

For miRNAs to be trafficked within MVs and perform biologically relevant roles when reaching their destinations, three conditions need to be fulfilled:

First: different populations of cells or tissues can produce MVs containing different and characteristic sets of miRNAs. There is growing evidence that the packaging of miRNAs into MVs can indeed exhibit tissue specificity (Braicu et al., 2015). For example, evidence to support the idea of a specific, controlled packaging mechanism for miRNAs has been reported in liver cells by Kogure et al. (2011). Similarly, MVs may also contain a non-random subset of miRNAs that occur within the parent cells as observed by Kogure et al. (2011), supporting the idea of specific packaging mechanisms. Many mammalian cells in culture retain the capacity to export miRNAs into the surrounding environment, which may explain the observation of high concentrations of miRNAs in fluids such as plasma (Wang et al., 2010). Though much more work is needed, in principle there is accumulating evidence that cells have the potential to package specific sets of miRNAs into MVs.
Second: $M V$ s are mobile and deliver their payload to potential target cells. RNAs can be transferred within MVs between immune mast cells in mouse and from mouse to human mast cells (Valadi et al., 2007). miRNA transfer in MVs from T cells to antigen presenting cells has also been shown (Mittlebrunn et al., 2011). In a different cellular context there is evidence that MVs released by male reproductive tissues in humans and in D. melanogaster fuse to sperm (Corrigan et al., 2014) and therefore have the potential to be transported to very specific places in the female-although it is not yet known whether these MVs contain miRNAs. To expand: in D. melanogaster, one of the two main cell types of the adult male accessory gland, the secondary cells (Figure 4), have been observed to secrete MVs (Corrigan et al. 2014), which, after mating fuse with sperm and can interact with the reproductive tract of the female. Indeed the prevention of the release of these MVs altered the normal inhibition of female sexual receptivity following mating.

Finally: the miRNAs delivered into cells can affect the expression of specific target genes. The final step is that miRNAs trafficked to specific cells in MVs need to initiate specific cell responses, that is, suppress the activity of specific mRNAs targeted by the miRNAs within the MVs. It is thought that miRNAs have the capacity to be delivered into target cells via fusion of MVs with the target plasma cell membrane or by endocytosis of MVs (reviewed in Weilner et al., 2013). Evidence that this can occur in principle comes mostly from in vitro experiments, particularly in the study of cancer and immune biology. The transfer of miRNA-bearing MVs between cell types has the potential to suppress target gene expression in the recipient cells (for example see Buck et al., 2014; Pegtel et al., 2014; Squadrito et al., 2014). For example, Skog et al. (2008) incorporated a reporter into MVs and showed that the RNAs trafficked within MVs (exosomes) from glioblastoma cancer cells into normal cells can be translated. The authors suggested that this may promote the formation of a tumourigenic environment (Skog et al., 2008). Another example occurs in liver cells in which MVs contain a highly enriched fraction of miRNAs in comparison to their parent cells, which can alter the expression of the TAK1 growth factor in recipient cells (Kogure et al., 2011). Similarly, again working in cell culture, Chen et al. (2014) showed evidence that chemotherapeutic resistance between breast cancer cell lines was associated with trafficking of miRNAs within MVs. The in vitro repression of target mRNAs in endothelial cells by miRNAs trafficked from macrophages has also been demonstrated (Squadrito et al., 2014). It is worth noting that episodic delivery of miRNAs in by MVs could contribute to considerable perturbation in gene expression.

miRNAs also have the potential to be trafficked in MVs between different species, though the extent and importance of this phenomenon is not yet known. Evidence to support the principle of this idea comes mostly from the study of immune biology. Proof of principle comes from the transfer and exchange of miRNAs between species, as shown in in vitro experiments in which nematode MVs containing miRNAs, YRNAs and an Argonaute protein were incubated with host mouse cells and showed suppression of gene expression of the genes targeted by the miRNAs (Buck et al., 2014). The suggestion that this is also possible in vivo is supported by the evidence from the same study in which miRNAs from a filarial nematode were found in the blood serum of infected mice (Buck et al., 2014).

The accumulating evidence shows that, in principle, miRNAs trafficked in MVs can serve as a mechanism of delivering genetic information. Increasing evidence supports the idea that MVs containing RNAs can be trafficked within individuals, between males and 


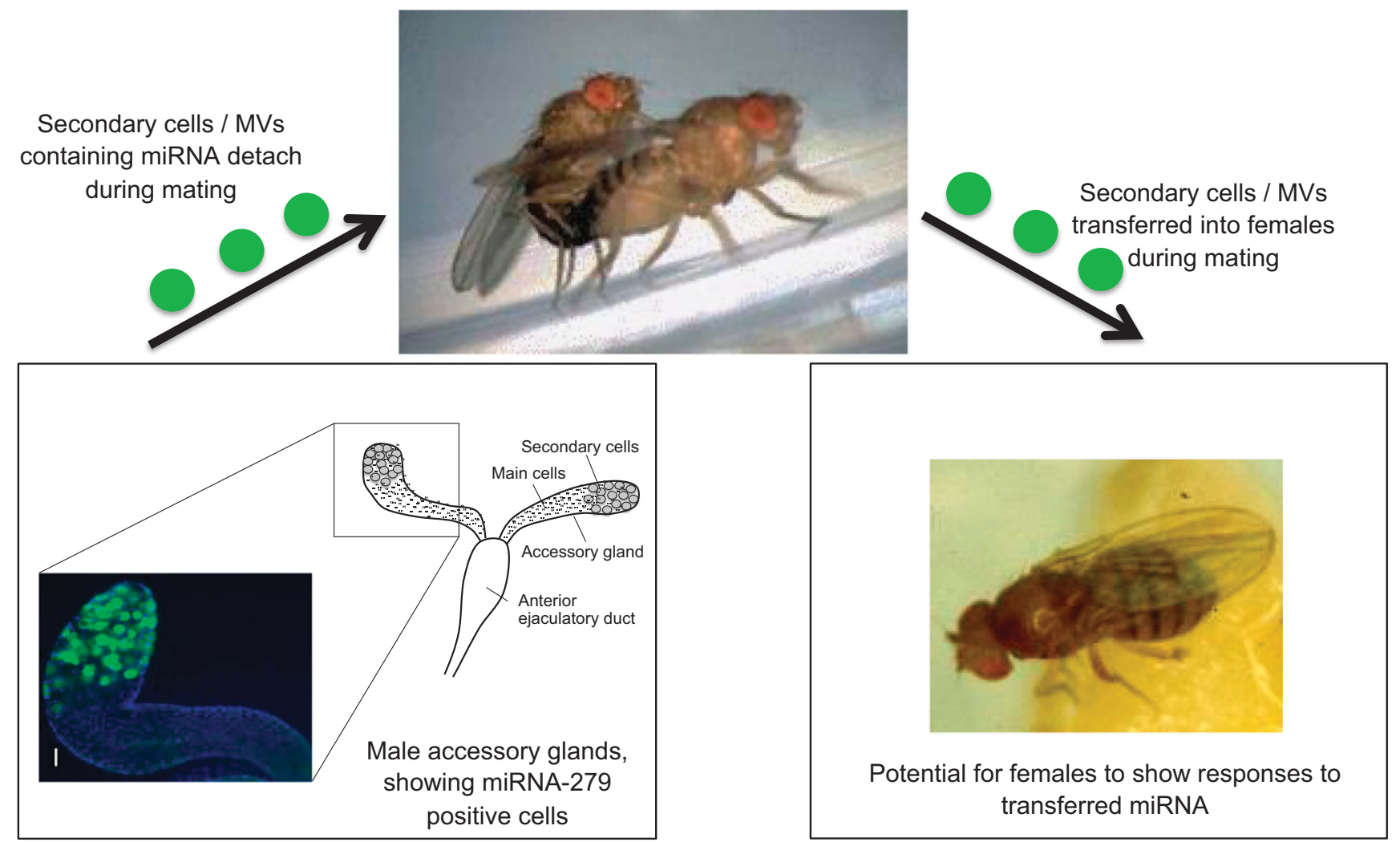

Figure $4 \mathrm{~A}$ hypothetical model for miRNA transfer between the sexes. Here, miR-279, which is implicated in microguarding against the costs of gene expression variation in females arising from sexual conflict (Fricke et al., 2014), has the potential to be transferred to females from males during mating. miR-279 is expressed in the secondary cells of the D. melanogaster male accessory gland (Green et al., unpublished data). The inset shows a confocal micrograph of the tip of an accessory gland of a 1 day-old male reproductive system, in a transgenic line in which the expression of miR-279 is tagged to a GFP reporter (miR-279-GAL4; UAS-GFP; Cayirlioglu et al., 2008). The bi-nucleate arrangement of the nuclei in the accessory gland main cells is evident by DAPI staining (blue). Scale bar, $50 \mu \mathrm{m}$. These secondary cells can detach and transfer across to females during mating (Leiblich et al., 2012). The secondary cells also release microvesicles (MVs) that are similarly transferred during mating and that can interact with female tissues and alter female post mating receptivity (Corrigan et al., 2014). This system therefore offers a potential model for miRNA trafficking between the sexes, in a process likely to be shaped by sexual conflict.

females (Corrigan et al., 2014) and even between species (for example, between parasites and their hosts; Barteneva et al., 2013; Buck et al., 2014). Horizontal transfer of miRNAs via MVs between tumour cells is reported and may represent a mechanism for conferring chemoresistance (Chen et al., 2014). miRNA-containing MVs have even been hypothesised as a mechanism for the transfer of epigenetic inheritance from germ line to soma (Sharma, 2014) and as potential contributors to mechanistic ageing (Weilner et al., 2013).

The major outstanding question is whether these effects occur to any extent in vivo and the relative role of the different types of MVs in this process (Bobrie et al., 2011). Even if the individual steps are all possible, we also need to know that all of them can occur efficiently in the same biological systems. The interesting possibility that MVs may contain biologically relevant sets of RNAs and other molecules that might act in concert has also hardly yet been considered.

\section{Conflict hypothesis for microguards and micromessengers}

Above we reviewed evidence for miRNAs as microguards of the genome to prevent gene expression variation with the potential to result in fitness costs, and the potential role of miRNAs as messengers that can themselves perturb gene expression in potentially remote target cells. These new functions for miRNAs are likely to frequently place them at the heart of interactions between different parties with different fitness interests. For example, cancer cells and the antecedents from which they are derived, males and females, hosts and disease-causing organisms often have divergent evolutionary interests because of their lack of genetic relatedness. Genes in cancer cells increase in frequency via proliferation and have little interest in promoting the survival of their parent, non-cancerous host cells, from which they become less and less related over time as they accrue mutations. Similarly, in promiscuous mating systems males often gain by forcing their unrelated mates to invest more in the current reproductive bout than may be optimal for the long-term interests of females. Finally, disease-causing organisms gain fitness from proliferation within hosts and effective spread to the next host, which is may often not be in the best reproductive strategy for the host itself.

These evolutionary tensions between interacting parties can often be a potent force for driving evolutionary change (Chapman et al., 2003a; Chapman, 2006). We explore these new ideas below and lay out a new hypothetical framework to highlight the role of conflict in shaping the evolution of microguards and micromessengers. Specifically we hypothesise that: (i) microguards may often function to dampen oscillations in gene frequency that arise precisely because of conflicts of interest; (ii) micromessengers may themselves be effective agents of conflict between different parties. It is clear that much more research 
Table 1 Predictions for biotic scenarios in which microguarding will confer significant fitness benefits

\begin{tabular}{|c|c|}
\hline Source & Source of gene expression variation and potential for microguarding \\
\hline Within individuals & $\begin{array}{l}\text { Degree of developmental stability: Mechanisms such as microguarding are expected to be important and efficient ways in which to carefully } \\
\text { fine-tune developmental processes, ensuring the correct balance between developmental signals. Perturbations to these signals will disrupt } \\
\text { normal development resulting in potentially significant fitness costs, selecting for any mechanisms that lower gene expression variation (Kim } \\
\text { et al., 2013). }\end{array}$ \\
\hline Within individual conflict & $\begin{array}{l}\text { Selfish genetic elements such as driving genes and transposable elements: relatively unregulated, and therefore potentially costly, gene } \\
\text { expression perturbations may be common whenever the genetic causative agent involved lacks fitness interests that are coincident with that of } \\
\text { the host in which it resides. Such a situation exists for selfish genetic elements that enhance their own transmission, sometimes at the expense } \\
\text { of their host genome. Hence if mechanisms by which such elements excise, replicate and increase in frequency result in costly gene expression } \\
\text { perturbation in the host, one outcome is selection for microguarding mechanisms. } \\
\text { Conflict within clones of cells within individuals: conflict is expected between different cancer clones within individuals or between cancer } \\
\text { clones and the hosts in which they reside. This has the potential to initiate unregulated gene expression variation and hence select for guarding } \\
\text { mechanisms, as above. }\end{array}$ \\
\hline
\end{tabular}

Sexual conflict

Sexual conflict

Conflict between males and females over reproductive decisions and shared reproductive traits: conflicts over, for example, the pattern of traits (such as mating frequency or reproductive investment) that are shared between males and females are common. For example, males may gain fitness by manipulating their mates to invest more than optimal and vice versa. An example is observed in the transfer of semen components from male to female fruit flies during mating that cause females to significantly increase their reproductive investment by laying more eggs. Such effects can lead to costs and widespread gene expression changes in females. Our recent data suggest that miRNA expression in females can reduce the costs of these effects (Fricke et al., 2014).

Sexual selection

Competition within and between the sexes: there is considerable variation in trait expression, and likely underlying gene expression, arising from male-male competition and from the expression of female mate choice. Costs arising from these competitions are also well known and could again select for gene expression dampening via microguards.

Parent offspring conflict Conflict between parents over the level of parental care: conflict over the level of provisioning across the placenta from mother to developing arising from asymmetries of relatedness between mother, father and offspring are well known. The embryo may gain from manipulating the mother into investing more resources than is optimal for her. On the basis of recent findings there is the potential for miRNAs to be messengers, as well as potential guardians of this conflict.

Hybridisation

Disruption of coadaptation via hybridisation: matings between different populations or locally adapted genotypes could generate gene expression fluctuation because of perturbation of co-adapted gene networks. Such matings are often observed to result in unpredictable outcomes and potential costs (for example see Andres and Arnqvist 2001) and if sufficiently frequent could select for microguarding as a mechanism to buffer costs.

Host-microorganism conflict Conflicts between hosts and the microorganisms that reside within them: the interests of hosts and their microbes are often not aligned. Success of the microorganisms may therefore often result in deleterious effects on the host, with selection for dampening mechanism such as microguarding.

is needed to test these hypotheses and we outline some of the approaches that could prove fruitful.

\section{Hypothesis: microguards evolve to dampen down conflicts of interest}

In general, we expect gene expression variation resulting in significant fitness costs to be minimised within individuals, because of selection for efficient homoeostasis. However, as noted above, whenever individuals encounter perturbations to gene expression from exogenous sources, there is the potential for microguarding to increase fitness and therefore to be strongly selected. This is of key importance when specific host responses are being targeted, as is often the case when there are conflicts of interest. The reason why conflicts are of particular relevance in this context is that it is in these situations that perturbations to gene expression will occur, precisely because the different parties may be trying to manipulate the other and force maladaptive over-investment patterns (Table 1). Potentially deleterious gene expression variation and fluctuation is almost a necessary outcome of this interaction.

Within individuals. We expect microguarding to evolve as a general surveillance mechanism to buffer against normal variation in homoeostatic processes (Kim et al., 2013). However, when the body encounters cells that have escaped homoeostatic control, for example, cancer cells that exhibit lower relatedness and increased heterogeneity in comparison with the rest of the cells in a tissue, then variation in mRNA levels is expected to increase and result in potentially maladaptive variation. Loss of homoeostatic control often accompanies the expression of mechanistic ageing. A recent review suggests that the release by cells of miRNA-filled MVs increases with age and that their resulting effects may be a contributory factor to the expression of proximate ageing (Weilner et al., 2013). Many studies investigating evolutionary theories of ageing centred on mutation accumulation (Medawar, 1952) have sought supporting evidence by testing for increased variation in trait expression with age. It would be useful to test this idea further and to examine whether loss of mechanisms to regulate gene expression variation, potentially including the loss of microguarding, are contributory factors. One further idea is that in the context of ageing it is possible that over an individual's lifetime cells could lose the ability to regulate the release of MVs (Weilner et al., 2013) or that target cells could lose the ability to buffer the effects of external perturbations such as those supplied by miRNAs delivered in MVs.

Between individuals of the same species. We predict that microguarding should be particularly prevalent in situations when there are 
conflicts of interest between the sexes. Such conflict has been well studied in fruit flies where it can be mediated via the actions of semen molecules transferred from males to females during mating (Chapman et al., 2003a). As noted above, miRNAs via their microguarding functions can protect individuals against the deleterious effects of gene expression variation that occur in response to mating and the receipt of seminal fluid molecules (Gioti et al., 2012; Fricke et al., 2014). The finding that miR-279 and miR-317, which can potentially mediate buffering functions in females, are normally significantly downregulated following mating (Fricke et al., 2014) suggests that males may currently have the upper hand in this conflict. Intriguingly, males potentially transfer miRNA-279 to females during mating, perhaps to give a 'compensatory dose'. Lower levels of miR-279 and miR-317 act to suppress microguarding and elevate offspring production, which may be deleterious for females in the longer term. The significant fitness costs that often arise because of male-female interactions (Chapman et al., 1995; 2003a; Arnqvist and Rowe, 2005; Chapman, 2006) appear to result, at least in part, due to the overactivation of reproductive processes in females. Hence mechanisms such as microguarding that can dampen and minimise such gene expression variation (Fricke et al. 2014) should be selected. That there is selectable genetic variation to minimise such costs is known (Wigby and Chapman, 2004) though the genetic basis of that response is as yet unclear.

Another context where there are often strong conflicts of interest between individuals of the same species are between parents and offspring. This can occur both before and after birth. For example, the maternal placenta-embryo axis is a site in which much conflict is predicted and indeed observed (Haig, 1993). This is because this site regulates the amount of provisioning that is directed towards the developing embryo from the maternal blood supply. Too little provisioning and the embryo suffers, too much and there may be costs to the mother (Haig, 1993). Of interest in terms of the involvement of miRNAs are recent findings that the placenta also releases MVs into the maternal blood supply during pregnancy (for example see, Sarker et al., 2014). Furthermore, placental-derived MVs are reported to contain miRNAs (Ouyang et al., 2014), which suggests a potential mechanism for the potential manipulation of the mother by the developing foetus.

Between individuals of different species. There are also often significant conflicts of interest between individuals of different species that directly interact and which may require buffering mechanisms to reduce costly outcomes. In the context of miRNA trafficking being a potential mechanism to mediate gene expression variation we are concerned with the intimate associations between hosts and the disease-causing organisms they harbour (Barteneva et al., 2013). However, as yet, there seems little evidence for microguarding in this context.

Overall, we expect microguarding whenever there is the possibility of unregulated gene expression with negative fitness consequences (Table 1). Microguarding is expected to be prevalent particularly when there are marked differences in the evolutionary interests (that is, conflict) of the interacting parties involved (Table 1) —which is a testable prediction. Future research could usefully search for microguarding under these specific scenarios.

\section{Hypothesis: micromessengers are agents of conflict between different interacting parties}

The potential for miRNAs to function as micromessengers to effect cell-to-cell communication at a distance represents considerable potential for the expression of conflicts of interest. For example, sexual conflict could be expressed if miRNAs delivered via MVs to a target cell in individuals of the opposite sex manipulated gene expression in a way that favours the fitness interests of the genes in the parent male cell over those of the recipient female cell. Below we suggest the various scenarios where conflicts could be expressed and describe evidence that is consistent with this hypothesis.

Within individuals. A potentially frequent example of conflicts of interest that could be mediated within individuals occurs between cancerous and non-cancerous cells within an individual. Cancerous cells that start to proliferate in an unregulated manner do so to further their own interests as they become genetically distinct from their surroundings (for example see Greaves, 2007). There are growing numbers of examples in which cancer cells show abnormal patterns of MV-associated miRNA export and trafficking. For example, there is correlative evidence that cancer cells can transfer chemoresistance horizontally between cells within hosts via miRNAs trafficked in MVs (Chen et al., 2014), though more direct evidence would be welcome. Ohshima et al. (2010) also report the selective export of the miRNA let-7 in MVs from a metastatic gastric cancer cell line, which was not observed in the control parental cell line. The export of the tumoursuppressive let-7 from the cancer cells is proposed to maintain their tumourigenic state and increase their fitness through further proliferation. Clearly much more research is required to discover the frequency and fitness consequences to cancer cells and non cancer cells of miRNA trafficking.

Between individuals of the same species. There is evidence of MV transfer from males to females during mating (Corrigan et al., 2014) and interactions of RNAs from those MVs with the female reproductive epithelium. The constituents of the MVs in this case are not yet known. But in principle, could be a mechanism for transferring genetic information from males to females during mating with the potential to exert biological effects (Figure 4). Though many more investigations are clearly needed, at least one miRNA, miR-279, which is implicated in microguarding, is expressed in the secondary cells of the male accessory glands (Figure 4) that produce the MVs described above. These cells can detach and be transferred to females during mating (Leiblich et al., 2012), Furthermore, the MVs released from the secondary cells of the male accessory glands within the male reproductive tract can fuse with sperm, be transferred during mating and interact with the female reproductive tract (Corrigan et al., 2014). Human MVs derived from the male prostate gland similarly fuse with sperm, suggesting that this phenomenon may be conserved across species. The receipt of human semen is known to induce the expression of immune genes in cells of the cervix (Sharkey et al., 2007), though whether there is any involvement of miRNAs in this process is as yet unknown. We conclude that data to support the hypothesis of miRNAs trafficked by MVs in conflicts of interest between the sexes are currently scant and further studies to document the biological roles of miRNAs transferred in MVs are sorely needed.

Between individuals of different species. Hosts and the disease-causing organisms they harbour rarely have coincident fitness interests (Chapman, 2006) and so the increasing reports of disease phenotypes being mediated via the transfer of miRNAs in MVs is of interest of the conflict framework we propose here. As noted above, miRNAs from a filarial nematode have been detected in mouse serum and in vitro the targeted suppression of two genes in mouse cells incubated with miRNA-containing MVs derived from a gastrointestinal nematode have been reported (Buck et al., 2014). In other cases, fungal 
pathogens have been observed to use small RNA transfer as a means to hijack and suppress host RNA silencing pathways, further expediting their invasion (Weiberg et al., 2013). These findings illustrate a mechanism by which parasites might manipulate their hosts and also demonstrate RNA transfer between species. Such trafficking between species may even extend across even wider taxonomic distances. For example, plant-derived miRNAs in human and porcine breast milk have been identified in silico (Lukasik and Zielenkiewicz, 2014). However, the functional significance of these findings remains to be determined.

\section{Antecedents of microguarding}

Variation in gene expression is likely an inevitable consequence of the mechanisms by which genes are regulated. However, whenever this variation is costly there will be selection for dampening mechanisms that could include microguards. In general we expect the tracking of gene expression by the mechanisms that control it to improve and become tighter over evolutionary time. These dynamics may also show differing patterns depending upon the relationship of the interacting parties, that is, the extent to which they are in evolutionary conflict. These ideas could in theory be tested in vivo in experimental evolution experiments via the creation of new pairings between miRNAs and targets by the creation of synthetic miRNAs or synthetic targets with engineered target seed sequences. This could be achieved by the genetic transformation of model organisms with engineered synthetic miRNAs or target sequences. It would also be of interest to explore whether there are qualitative differences in gene expression variation in different contexts. Future work will show whether microguarding provides a more or less tight control of gene expression variation to be deployed in certain contexts over others. When greater numbers of examples of microguards have been identified phylogenetic investigations of the evolution of microguards versus their target genes could also be informative in picking apart the evolutionary sequence of events involved.

\section{SUMMARY}

The study of RNA silencing, originating from experiments in animals such as C. elegans and D. melanogaster and plants such as Petunia hybrida and Arabidopsis thaliana, continues to uncover surprising and novel functions of regulatory RNAs. Microguarding-mediated by miRNAs-appears to be a fast-acting response to exogenously induced antagonistic gene fluctuations. This represents an additional facet of small RNA regulation in addition to the already known roles in longterm homoeostatic gene regulation and micromanaging (Bartel and Chen, 2004). The fitness costs of defective microguarding are predicted to result in accelerated ageing and increased disposition to infectious disease. Further studies are now needed to explore this concept and to identify further examples of microguarding. The reduction of ligation bias in genome-wide small RNA cloning for next generation sequencing is uncovering new classes of small RNA that were not previously considered (Xu et al., 2015). We may uncover new miRNAs or other types of small RNA with a greater influence on microguarding. Our review has defined scenarios under which we expect microguarding to be prevalent, which may help focus the search for new examples. We also explored the significance of recent discoveries of trafficking of MVs containing miRNAs. Cells can release MVs whose contents exhibit some cell or tissue specificity, that can be delivered to specific target cells or tissues and that can in principle alter the expression of specific target genes in different tissues, individuals or even different species. In this way miRNAs can be part of a mechanism to both regulate and perturb gene expression.
We concluded by setting out two hypotheses in which we expect miRNAs to have significant roles in buffering against the effects of, or being the agents of, conflicts of interest. The scant evidence so far is consistent with these hypotheses but it is clear that many more data are needed before these ideas can be fully evaluated. Further exploration of the microguarding and micromessenger phenomena in model systems such as D. melanogaster and C. elegans could be especially informative. In a continuation of the evolutionary arms race between the sexes, males may transfer miRNAs or other small RNAs in their ejaculate during mating to disrupt or complement female microguarding that would otherwise jeopardise male mating success. The use of this system with its powerful experimental tractability may give clues to the significance of miRNA-containing MVs.

\section{DATA ARCHIVING}

There were no data to deposit.

\section{CONFLICT OF INTEREST}

The authors declare no conflict of interest.

\section{ACKNOWLEDGEMENTS}

We thank the Big $\mathrm{C}$ and the Biotechnology and Biological Sciences Research Council (grants BB/H008047/1 and BB/L003139/1) for funding and the Editor and three anonymous reviewers for their constructive comments.

Andres JA, Arnqvist G (2001). Genetic divergence of the seminal signal-receptor system in houseflies: the footprints of sexually antagonistic coevolution? Proc Biol Sci 268 399-405.

Arnqvist G, Rowe L (2005). Sexual Conflict. Princeton University Press: Princeton, NJ, USA.

Bartel DP, Chen CZ (2004). Micromanagers of gene expression: the potentially widespread influence of metazoan microRNAs. Nat Rev Genet 5: 396-400.

Barteneva NS, Maltsev N, Vorobjev IA (2013). Microvesicles and intercellular communication in the context of parasitism. Front Cell Infect Microbio/ 3: 49.

Bobrie A, Colombo M, Raposo G, Thery C (2011). Exosome secretion: molecular mechanisms and roles in immune responses. Traffic 12: 1659-1668.

Braicu C, Tomuleasa C, Monroig P, Cucuianu A, Berindan-Neagoe I, Calin GA (2015). Exosomes as divine messengers: are they the Hermes of modern molecular oncology? Cell Death Differ 22: 34-45.

Buck AH, Coakley G, Simbari F, McSorley HJ, Quintana JF, Le Bihan T et al. (2014). Exosomes secreted by nematode parasites transfer small RNAs to mammalian cells and modulate innate immunity. Nat Commun 5: 5488.

Cayirlioglu P, Kadow IG, Zhan X, Okamura K, Suh GS, Gunning D et al. (2008). Hybrid neurons in a microRNA mutant are putative evolutionary intermediates in insect $\mathrm{CO} 2$ sensory systems. Science 319: 1256-1260.

Chapman T (2006). Evolutionary conflicts of interest between males and females. Curr Biol 16: 744-754.

Chapman T, Arnqvist G, Bangham J, Rowe L (2003a). Sexual conflict. Trends Ecol Evol 18: 41-47.

Chapman T, Bangham J, Vinti G, Seifried B, Lung O, Wolfner MF et al. (2003b). The sex peptide of Drosophila melanogaster: female post-mating responses analyzed by using RNA interference. Proc Natl Acad Sci USA 100: 9923-9928.

Chapman T, Liddle LF, Kalb JM, Wolfner MF, Partridge L (1995). Cost of mating in Drosophila melanogaster females is mediated by male accessory gland products. Nature 373: 241-244.

Chen X, Ba Y, Ma L, Cai X, Yin Y, Wang K et al. (2008). Characterization of microRNAs in serum: a novel class of biomarkers for diagnosis of cancer and other diseases. Cell Res 18: 997-1006.

Chen WX, Cai YQ, Lv MM, Chen L, Zhong SL, Ma TF et al. (2014). Exosomes from docetaxel-resistant breast cancer cells alter chemosensitivity by delivering microRNAs. Tumour Biol 35: 9649-9659.

Chevillet JR, Kang Q, Ruf IK, Briggs HA, Vojtech LN, Hughes SM et al. (2014). Quantitative and stoichiometric analysis of the microRNA content of exosomes. Proc Natl Acad Sci USA 111: 14888-14893.

Corrigan L, Redhai S, Leiblich A, Fan SJ, Perera SM, Patel R et al. (2014). BMP-regulated exosomes from Drosophila male reproductive glands reprogram female behavior. J Cell Biol 206: 671-688.

Dalmay T (2008). MicroRNAs and cancer. J Intern Med 263: 366-375.

Filipowicz W, Bhattacharyya SN, Sonenberg N (2008). Mechanisms of post-transcriptional regulation by microRNAs: are the answers in sight? Nat Rev Genet 9: 102-114. 
Fricke C, Green D, Smith D, Dalmay T, Chapman T (2014). MicroRNAs influence reproductive responses by females to male sex peptide in Drosophila melanogaster. Genetics 198: 1603-1619.

Ghildiyal M, Zamore PD (2009). Small silencing RNAs: an expanding universe. Nat Rev Genet 10: 94-108.

Gioti A, Wigby S, Wertheim B, Schuster E, Martinez P, Pennington CJ et al. (2012). Sex peptide of Drosophila melanogaster males is a global regulator of reproductive processes in females. Proc Biol Sci 279: 4423-4432.

Greaves M (2007). Darwinian medicine: a case for cancer. Nat Rev Cancer 7: 213-221.

Hager GL, McNally JG, Misteli T (2009). Transcription dynamics. Mol Cell 35: 741-753.

Haig D (1993). Genetic conflicts in human pregnancy. Q Rev Biol 68: 495-532.

Hall AE, Dalmay T (2013). Discovery of novel small RNAs in the quest to unravel genome complexity. Biochem Soc Trans 41: 866-870.

Kim VN, Han J, Siomi MC (2009). Biogenesis of small RNAs in animals. Nat Rev Mol Cell Biol 10: 126-139.

Kim D, Grun D, van Oudenaarden A (2013). Dampening of expression oscillations by synchronous regulation of a microRNA and its target. Nat Genet 45: 1337-1344.

Kogure T, Lin W-L, Yan IK, Braconi C, Patel T (2011). Intercellular nanovesicle-mediated microRNA transfer: a mechanism of environmental modulation of hepatocellular cancer cell growth. Hepatology 54: 1237-1248.

Kumar P, Anaya J, Mudunuri SB, Dutta A (2014). Meta-analysis of tRNA derived RNA fragments reveals that they are evolutionarily conserved and associate with AGO proteins to recognize specific RNA targets. BMC Biol 12: 78.

Lawniczak MK, Begun DJ (2004). A genome-wide analysis of courting and mating responses in Drosophila melanogaster females. Genome 47: 900-910.

Lee Y, Kim M, Han J, Yeom KH, Lee S, Baek SH et al. (2004). MicroRNA genes are transcribed by RNA polymerase II. EMBO J 23: 4051-4060.

Leiblich A, Marsden L, Gandy C, Corrigan L, Jenkins R, Hamdy F et al. (2012). Bone morphogenetic protein- and mating-dependent secretory cell growth and migration in the Drosophila accessory gland. Proc Natl Acad Sci USA 109: 19292-19297.

Liu H, Kubli E (2003). Sex-peptide is the molecular basis of the sperm effect in Drosophila melanogaster. Proc Natl Acad Sci USA 100: 9929-9933.

Lotvall J, Valadi H (2007). Cell to cell signalling via exosomes through esRNA. Cell Adh Migr 1: 156-158.

Lukasik A, Zielenkiewicz P (2014). In silico identification of plant miRNAs in mammalian breast milk exosomes-a small step forward? PLoS One 9: e99963.

Marin-Gonzalez E, Suarez-Lopez P (2012). And yet it moves: cell-to-cell and long-distance signaling by plant microRNAs. Plant Sci 196: 18-30.

Mathivanan S, Ji H, Simpson RJ (2010). Exosomes: extracellular organelles important in intercellular communication. J Proteomics 73: 1907-1920.

McGraw LA, Gibson G, Clark AG, Wolfner MF (2004). Genes regulated by mating, sperm, or seminal proteins in mated female Drosophila melanogaster. Curr Biol 14: 1509-1514.

McGraw LA, Clark AG, Wolfner MF (2008). Post-mating gene expression profiles of female Drosophila melanogaster in response to time and to four male accessory gland proteins. Genetics 179: 1395-1408.

Medawar PB (1952). An Unsolved Problem In Biology. HK Lewis: London, UK.

Mitchell PS, Parkin RK, Kroh EM, Fritz BR, Wyman SK, Pogosova-Agadjanyan EL et al. (2008). Circulating microRNAs as stable blood-based markers for cancer detection. Proc Natl Acad Sci USA 105: 10513-10518.

Mittelbrunn M, Guttiérrez-Vázquez C, Villarroya-Beltri C, González S, Sánchez-Cabo F, González MA et al. (2011). Unidirectional transfer of microRNA-loaded exosomes from T cells to antigen-presenting cells. Nat Commun 2: 282.

Ohshima K, Inoue K, Fujiwara A, Hatakeyama K, Kanto K, Watanabe Y et al. (2010). Let-7 microRNA family is selectively secreted into the extracellular environment via exosomes in a metastatic gastric cancer cell line. PLoS One 5: e13247.

Ouyang Y, Mouillet JF, Coyne CB, Sadovsky Y (2014). Placenta-specific microRNAs in exosomes-good things come in nano-packages. Placenta 35: S69-S73.

Parent JS, Martinez de Alba AE, Vaucheret $H$ (2012). The origin and effect of small RNA signaling in plants. Front Plant Sci 3: 179

Pegtel DM, Peferoen L, Amor S (2014). Extracellular vesicles as modulators of cell-to-cell communication in the healthy and diseased brain. Philos Trans $R$ Soc Lond B Biol Sci 369: 20130516.

Perkel JM (2013). Visiting 'noncodarnia'. BioTechniques 54: 303-304.

Rechavi O, Houri-Ze'evi L, Anava S, Goh WS, Kerk SY, Hannon GJ et al. (2014). Starvationinduced transgenerational inheritance of small RNAs in C. elegans. Cell 158: 277-287.
Sarker S, Scholz-Romero K, Perez A, Illanes SE, Mitchell MD, Rice GE et al. (2014). Placenta-derived exosomes continuously increase in maternal circulation over the first trimester of pregnancy. J Trans/ Med 12: 204.

Skog J, Würdinger T, van Rijn S, Meijer DH, Gainche L, Curry WT Jr et al. (2008). Glioblastoma microvesicles transport RNA and proteins that promote tumour growth and provide diagnostic biomarkers. Nat Cell Biol 10: 1470-1476.

Sharkey DJ, Macpherson AM, Tremellen KP, Robertson SA (2007). Seminal plasma differentially regulates inflammatory cytokine gene expression in human cervical and vaginal epithelial cells. Mol Hum Reprod 13: 491-501.

Sharma A (2014). Bioinformatic analysis revealing association of exosomal mRNAs and proteins in epigenetic inheritance. J Theor Biol 357: 143-149.

Sirot LK, Wong A, Chapman T, Wolfner MF (2014). Sexual conflict and seminal fluid proteins: a dynamic landscape of sexual interactions. In: Sexual Conflict. EdsRice WR, Gavrilets S. Cold Spring Harbor Laboratory Press: Cold Spring Harbor, NY, USA.

Squadrito ML, Baer C, Burdet F, Maderna C, Gilfillan GD, Lyle R et al. (2014). Endogenous RNAs modulate microRNA sorting to exosomes and transfer to acceptor cells. Cell Rep 8: $1432-1446$.

Sun K, Jee D, de Navas LF, Duan H, Lai EC (2015). Multiple in vivo biological processes are mediated by functionally redundant activities of Drosophila mir-279 and mir-996. PLoS Genet 11: e1005245.

Swingler TE, Wheeler G, Carmont V, Elliott HR, Barter MJ, Abu-Elmagd M et al. (2012). The expression and function of microRNAs in chondrogenesis and osteoarthritis. Arthritis Rheum 64: 1909-1919.

Tili E, Michaille JJ, Wernicke D, Alder H, Costinean S, Volinia S et al. (2011). Mutator activity induced by microRNA-155 (miR-155) links inflammation and cancer. Proc Natl Acad Sci USA 108: 4908-4913.

Valadi H, Ekström K, Bossios A, Sjöstrand M, Lee JJ, Lötvall JO (2007). Exosome-mediated transfer of mRNAs and microRNAs is a novel mechanism of genetic exchange between cells. Nat Cell Biol 9: 654-659.

Vojtech L, Woo S, Hughes S, Levy C, Ballweber L, Sauteraud RP et al. (2014). Exosomes in human semen carry a distinctive repertoire of small non-coding RNAs with potential regulatory functions. Nucleic Acids Res 42: 7290-7304.

Wang K, Zhang S, Weber J, Baxter D, Galas DJ (2010). Export of microRNAs and microRNA-protective protein by mammalian cells. Nucleic Acids Res 38: 7248-7259.

Weiberg A, Wang M, Lin F-M, Zhao H, Zhang Z, Kaloshian I et al. (2013). Fungal small RNAs suppress plant immunity by hijacking host RNA interference pathways. Science 342: 118-123.

Weilner S, Schraml E, Redl H, Grillari-Voglauer R, Grillari J (2013). Secretion of microvesicular miRNAs in cellular and organismal aging. Exp Gerontol 48: 626-633.

Wigby S, Chapman T (2004). Female resistance to male harm evolves in response to manipulation of sexual conflict. Evolution 58: 1028-1037.

Wigby S, Chapman T (2005). Sex peptide causes mating costs in female Drosophila melanogaster. Curr Biol 15: 316-321.

Xu P, Billmeier M, Mohorianu I, Green D, Fraser WD, Dalmay T (2015). An improved protocol for small RNA library construction using high definition adapters. Methods Next Gen Seq 2: 1-10.

Yoo B-C, Kragler F, Varkonyi-Gasic E, Haywood V, Archer-Evans S, Lee YM et al. (2004). A systemic small RNA signaling system in plants. Plant Cell 16: 1979-2000.

Zhou H, Rigoutsos I (2014). MiR-103a-3p targets the 5' UTR of GPRC5A in pancreatic cells. RNA 20: 1431-1439.

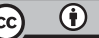

This work is licensed under a Creative Commons Attribution 4.0 International License. The images or other third party material in this article are included in the article's Creative Commons license, unless indicated otherwise in the credit line; if the material is not included under the Creative Commons license, users will need to obtain permission from the license holder to reproduce the material. To view a copy of this license, visit http:// creativecommons.org/licenses/by/4.0/ 